University of Nebraska - Lincoln

DigitalCommons@University of Nebraska - Lincoln

2012

\title{
L10 CrPt phase formation and magnetic properties
}

\author{
Rui Zhang \\ University of Nebraska-Lincoln \\ Ralph Skomski \\ University of Nebraska-Lincoln, rskomski2@unl.edu \\ Xingzhong $\mathrm{Li}$ \\ University of Nebraska - Lincoln, xli2@unl.edu \\ Zhen Li \\ University of Nebraska-Lincoln \\ Priyanka Manchanda \\ LMN Institute of Information Technology, priyanka.manchanda@vanderbilt.edu \\ See next page for additional authors
}

Follow this and additional works at: https://digitalcommons.unl.edu/physicssellmyer

Part of the Physics Commons

Zhang, Rui; Skomski, Ralph; Li, Xingzhong; Li, Zhen; Manchanda, Priyanka; Kashyap, Arti; Kirby, Roger D.; Liou, Sy-Hwang; and Sellmyer, David J., "L10 CrPt phase formation and magnetic properties" (2012). David Sellmyer Publications. 235.

https://digitalcommons.unl.edu/physicssellmyer/235

This Article is brought to you for free and open access by the Research Papers in Physics and Astronomy at DigitalCommons@University of Nebraska - Lincoln. It has been accepted for inclusion in David Sellmyer Publications by an authorized administrator of DigitalCommons@University of Nebraska - Lincoln. 


\section{Authors}

Rui Zhang, Ralph Skomski, Xingzhong Li, Zhen Li, Priyanka Manchanda, Arti Kashyap, Roger D. Kirby, SyHwang Liou, and David J. Sellmyer 


\title{
$L 1_{0}$ CrPt phase formation and magnetic properties
}

\author{
Rui Zhang, ${ }^{1, a)}$ Ralph Skomski, ${ }^{1}$ Xingzhong Li, ${ }^{1}$ Zhen Li, ${ }^{1}$ Priyanka Manchanda, ${ }^{2}$ \\ Arti Kashyap, ${ }^{2}$ Roger D. Kirby, ${ }^{1}$ Sy-Hwang Liou, ${ }^{1}$ and D. J. Sellmyer ${ }^{1}$ \\ ${ }^{1}$ Department of Physics \& Astronomy and Nebraska Center for Materials and Nanoscience, \\ University of Nebraska, Lincoln, Nebraska 68588, USA \\ ${ }^{2}$ LNM Institute of Information Technology, Jaipur, Rajasthan, India
}

(Presented 3 November 2011; received 23 September 2011; accepted 23 November 2011; published online 8 March 2012)

\begin{abstract}
$L 1_{0}$-ordered antiferromagnetic $\mathrm{CrPt}$ is of interest as a pinning material in exchange-biased system due to its many intriguing properties and such alloy with a (001) texture has also been used to serve as an underlayer to promote the $L 1_{0}$ phase formation of other materials. Therefore, it is important to control not only the $L 1_{0}$ phase formation of such material but also the texture of its ordered phase. A systematic study of the $L 1_{0}$ phase formation of $\mathrm{CrPt}$ thin film was performed. The anisotropy of $\mathrm{CrPt} L 1_{0}$ phase has also been investigated both experimentally using $\mathrm{CrPt} / \mathrm{Fe}$ bilayer system and theoretically using first principle calculation. The experimental result is in consistent with the theoretical estimation within the present thin film limitation. (C) 2012 American Institute of Physics. [doi:10.1063/1.3677928]
\end{abstract}

\section{INTRODUCTION}

Antiferromagnetic $L 1_{0}$-ordered $\mathrm{CrPt}$ is of interest as a pinning material in exchange-biased systems due to its high blocking temperature, simple hysteresis loops, and high corrosion resistance. Furthermore, $\mathrm{Cr}$ undergoes much less harmful interdiffusion compared to $\mathrm{Mn}$ atoms in Mn-based alloys during heat treatments. ${ }^{1}$ Moreover, thin films of $L 1_{0}$ phase $\mathrm{CrPt}$ with a (001) texture have been used as an underlayer to promote the $L 1_{0}$ phase formation of subsequent materials such as $\mathrm{FePt}{ }^{2}$ which is a promising candidate for future recording media. ${ }^{3,4}$ It is important to control and understand both formation of the $L 1_{0}$ phase and the texture with which it grows.

In addition to the aforementioned practical applications of $L 1_{0}$ phase $\mathrm{CrPt}$, the spin structure and micromagnetism of this intriguing system is not fully understood, especially its anisotropy. Figure 1 shows the schematic crystal structure and spin configuration of $L 1_{0} \mathrm{CrPt}$ based on neutron diffraction. ${ }^{5}$ Each $\mathrm{Cr}$ atom carries $2.24 \pm 0.15 \mu_{\mathrm{B}}$ moments, which is antiferromagnetically aligned with its nearest neighbors in the (001) planes while the contribution from $\mathrm{Pt}$ is extremely small. ${ }^{5,6}$ Preliminary research into the anisotropy has led to an experimental estimate of $10 \mathrm{~kJ} / \mathrm{m}^{3}$ by measuring the exchange bias in an $\mathrm{FeCo} / \mathrm{CrPt}$ bilayer system. ${ }^{1}$ This method may not be accurate due to its incomplete exchange coupling between the bilayers, which may mimic a strongly reduced anisotropy.

This paper reports a systematic study of the effect of deposition and processing conditions on $L 1_{0}$ phase formation in CrPt thin films and an investigation of the resulting anisotropy using magnetic measurements of an exchange-coupled $\mathrm{CrPt} / \mathrm{Fe}$ system as well as first-principle calculations.

\section{EXPERIMENTAL DETAILS}

Three series of $40 \mathrm{~nm}$ CrPt samples (labeled A, B, and $\mathrm{C}$ ) were prepared on thermally oxidized $\mathrm{Si}$ substrates

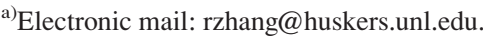

using magnetron sputtering at a base pressure of $10^{-8}$ Torr. Series A samples were deposited at room temperature (RT) by co-sputtering from pure $\mathrm{Cr}$ and $\mathrm{Pt}$ targets. Series $\mathrm{B}$ was deposited at $\mathrm{RT}$ in a multilayer structure of $[\operatorname{Cr}(x \AA) /$ $\operatorname{Pt}(1.7 \AA)]_{n}$ where $x$ was tuned using EDX to achieve $50 / 50$ composition. Sample series $\mathrm{C}$ was deposited at temperatures ranging from $300{ }^{\circ} \mathrm{C}$ to $800^{\circ} \mathrm{C}$ using co-sputtering. An additional co-sputtered sample deposited at $600{ }^{\circ} \mathrm{C}$ was capped at RT with a Fe layer whose thickness ranged from 4 to $9 \mathrm{~nm}$. Samples from series A and B were annealed by either rapid thermal annealing (RTA) or conventional furnace (CF) in $\mathrm{H}_{2} /$ Ar forming gas for $5 \mathrm{~min}$ and $5 \mathrm{~h}$, respectively.

The phase formation and texture of $\mathrm{CrPt}$ were characterized by an X-ray diffractometer, and a transmission electron microscope (TEM) while the magnetic measurements were performed using an alternating gradient force magnetometer (AGFM), and a superconducting quantum interference device (SQUID) magnetometer.

\section{RESULTS AND DISCUSSION}

X-ray diffraction spectra are shown in Fig. 2 for series $\mathrm{A}$ and $\mathrm{B}$ samples processed at different temperatures in the CF. The as-deposited samples show strong (111) texture. The shift of this peak to a lower angle upon annealing indicates the formation of the $L 1_{0}$ phase, which has a larger lattice spacing along the body diagonal. Only after annealing above $500{ }^{\circ} \mathrm{C}$ does the $L 1_{0}$ phase (001) peak appear along with the $(200) /(002)$ peak and the intensity of those peaks becomes quite pronounced as the annealing temperature increases. These XRD patterns indicate that the $L 1_{0}$ phase formation for post-deposition annealed co-sputtered films, series A, and multilayer films, series B, follow a similar trend. However, the degree of crystallinity, as estimated by peak intensities, in series A appears to be higher than that in series B for any given annealing temperature. Similarly, the degree of $L 1_{0}$ order and (001) texture appears more prevalent 


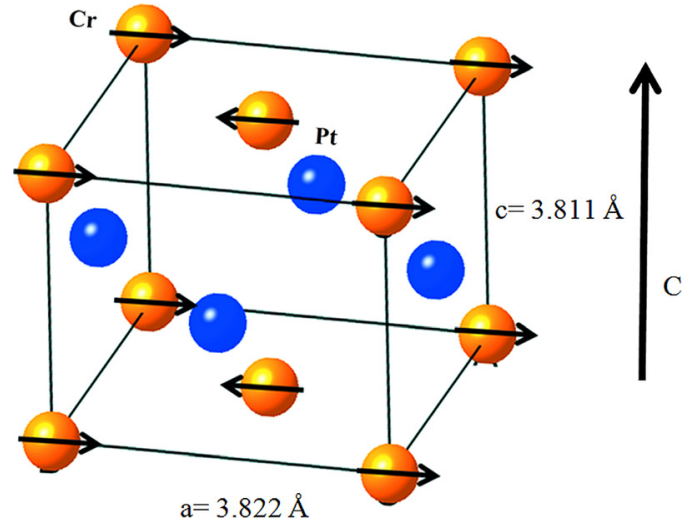

FIG. 1. (Color online) Schematic crystal structure and spin configuration of $L 1_{0} \mathrm{CrPt}$. The preferential magnetization direction is in the basal plane, corresponding to a negative anisotropy constant $K_{1}$.

in series A than in series B. Although similar trend can be found for samples annealed using RTA (not shown here), this difference is most prevalent in the samples annealed under CF. Unlike FePt, the stack structure did not seems to help promoting the [001] texture of $L 1_{0}$ phase CrPt. This is likely because the interdiffusion between $\mathrm{Cr}$ and $\mathrm{Pt}$ is very difficult and only occurs at temperature above $500{ }^{\circ} \mathrm{C} .{ }^{7}$

In the series of samples deposited at elevated temperatures, those deposited below $500^{\circ} \mathrm{C}$ show strong out-ofplane (111) texture [Fig. 3(a)]. Although the (001) peak is present in samples deposited above $600^{\circ} \mathrm{C}$, with an intensity increasing with temperature, the ratios with the (002) and (111) peaks do not indicate a high degree of $L 1_{0}$ order or (001) texture, respectively. A selected-area-electrondiffraction (SAED) pattern was taken on the sample deposited at $600{ }^{\circ} \mathrm{C}$ [Fig. 3(b)]. A calculated polycrystalline pattern was produced using the PCED2.0 program, ${ }^{8}$ in which the (001) texture is simulated based on the March model. Within this model, crystalline texture is quantified by the parameter $r$, where $r=0$ corresponds to perfect texture and $r=1$ for fully random orientation. The March parameter for this sample is estimated to be $r=0.65$. This result suggests that roughly $60 \%$ of the $c$-axis of $\mathrm{CrPt}$ is within $60^{\circ}-90^{\circ}$ of the film plane for this particular sample.

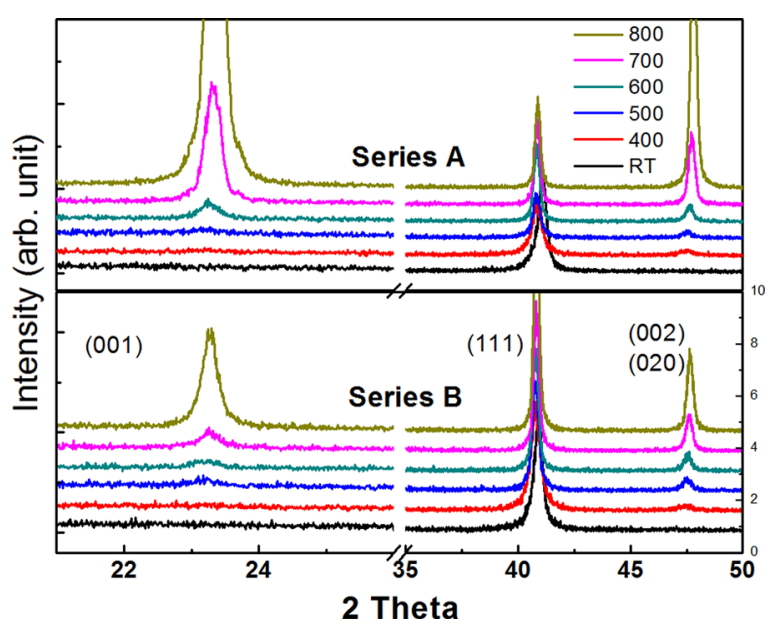

FIG. 2. (Color online) XRD measurement of annealed sample series A and $\mathrm{B}$ using $\mathrm{CF}$.
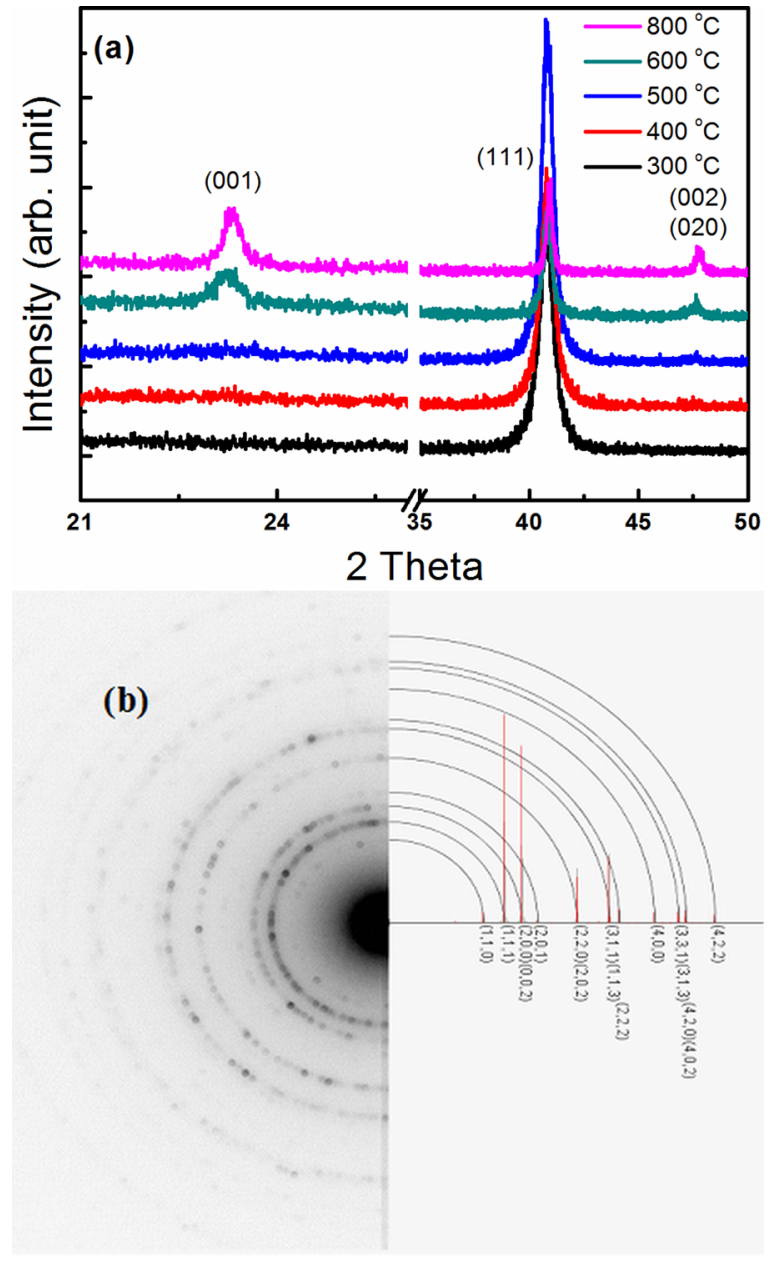

FIG. 3. (Color online) (a) XRD measurement of series C; (b) Experimental SAED pattern of the sample deposited at $600{ }^{\circ} \mathrm{C}$ and simulated ED pattern.

The in-plane hysteresis loops [Fig. 4(a)] of $\mathrm{CrPt} / \mathrm{Fe}$ bilayer system measured by AGFM show increasing coercivity $\left(H_{c}\right)$ from around 12 to $42 \mathrm{mT}$ and are nearly rectangular until the film thickness drops below $5 \mathrm{~nm}$, where they become twophase like transition. Perpendicular hysteresis loop could not be saturated until $2 \mathrm{~T}$ and a two-phase transition in hysteresis loop can be observed [Fig. 4(b)] for Fe with thickness higher than $6 \mathrm{~nm}$ below which it turns to a straight line. The hysteretic field or "coercivity" $H_{c}$ of these hysteresis loops varies from 0.12 to $0.28 \mathrm{~T}$ as the thickness of $\mathrm{Fe}$ decreases.

Figure 4 shows that the $\mathrm{CrPt}$ exhibits a substantial anisotropy, much more than the previously estimated anisotropy constant of $10 \mathrm{~kJ} / \mathrm{m}^{3}$. An estimate for the anisotropy is obtained by equating $H_{c}$ in Fig. 4(b) with the anisotropy field $H_{A}$. Since the magnetization and the anisotropy originate nearly exclusively from the $\mathrm{Fe}$ and the $\mathrm{CrPt}$, respectively, we can write the anisotropy field as

$$
H_{\mathrm{A}}=\frac{2 K_{\mathrm{CrPt}} t_{\mathrm{CrPt}}}{\mu_{\mathrm{o}} M_{\mathrm{Fe}} t_{\mathrm{Fe}}} .
$$

Using $\mu_{\mathrm{o}} H_{c}=0.28 \mathrm{~T}, t_{\mathrm{CrPt}}=40 \mathrm{~nm}$, and $t_{\mathrm{Fe}}=7 \mathrm{~nm}$ yields $K_{\mathrm{CrPt}}=-438 \mathrm{~kJ} / \mathrm{m}^{3}$. Thicker Fe layers yield less reliable estimates, because the Fe magnetization gets more and more inhomogeneous as the Fe thickness increases, and this effect is not included in Eq. (1). 

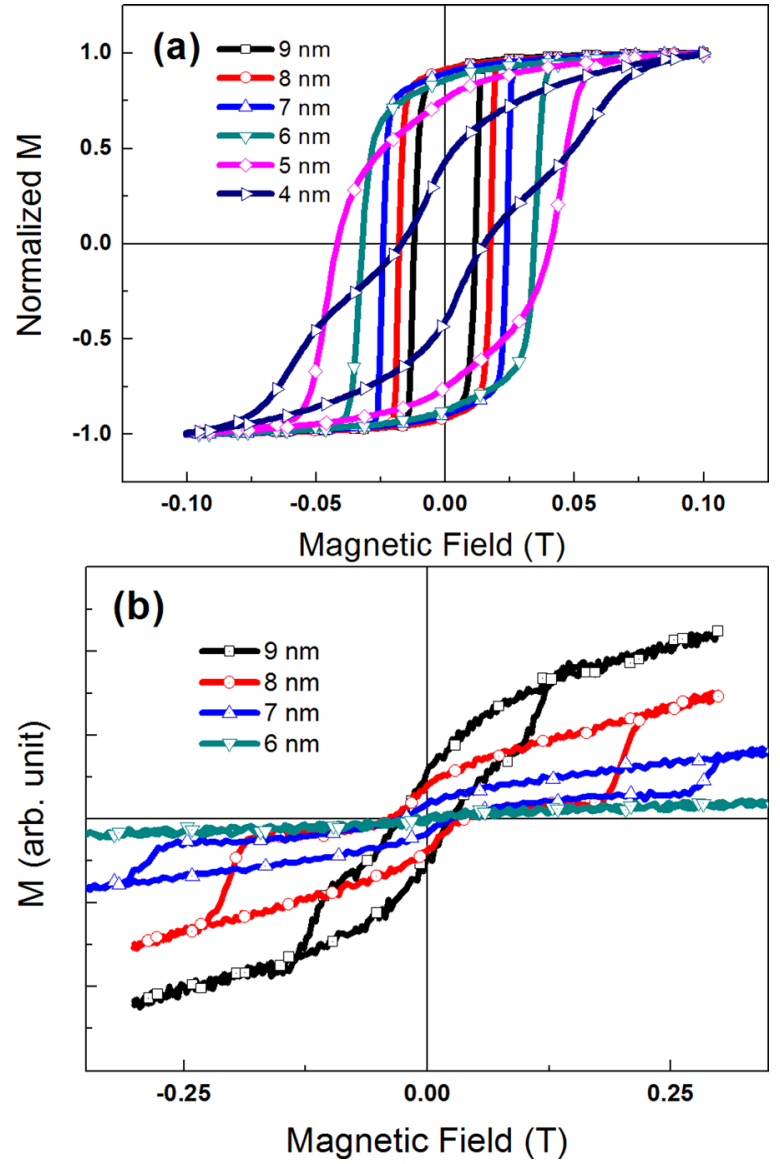

FIG. 4. (Color online) In-plane (a) and perpendicular (b) hysteresis loop of $\mathrm{CrPt} / \mathrm{Fe}$ measured by AGFM.

To theoretically investigate the anisotropy, we have performed a first-principle calculations of the first uniaxial $\mathrm{CrPt}$ anisotropy constant $K_{1}$. The ab-initio calculations were performed by using the projected augmented wave method and the generalized gradient approximation (GGA) for exchange and correlation, as implemented by the Vienna Ab-initio Simulation Package. 9 The magnetic anisotropy constant is achieved by performing the calculations in (100) magnetization direction and (001) direction in the of presence spinorbit coupling. The calculated value of magnetic anisotropy constant is $K_{l}=-3500 \mathrm{~kJ} / \mathrm{m}^{3}$, and this anisotropy constant is quite robust against competing types of AFM order. The negative sign of the anisotropy is in agreement with the observation of easy-plane anisotropy of $L 1_{0}$ phase $\mathrm{CrPt}$ using neutron spectroscopy. 5

The experimental coercivities are generally much smaller than the anisotropy field, often by one or two orders of magnitude. ${ }^{10}$ This is because Eq. (1) assumes coherent rotation, whereas coercivities encountered in practice are generally strongly reduced by incoherent magnetization processes. ${ }^{10,11}$ Moreover, the texture analysis above shows that a substantial fraction of the grains is misaligned with respect to the $c$-axis. The mechanism of Fig. 5 illustrates how $c$-axis misalignment translates into coercivity, even if $K_{1}<0$ which led to the hysteresis loops of Fig. 4(b). This micromagnetic feature contributes to the further reduction of $H_{c}$. For these reasons, we expect the true anisotropy and the true anisotropy constant to be much higher than the experimental value.

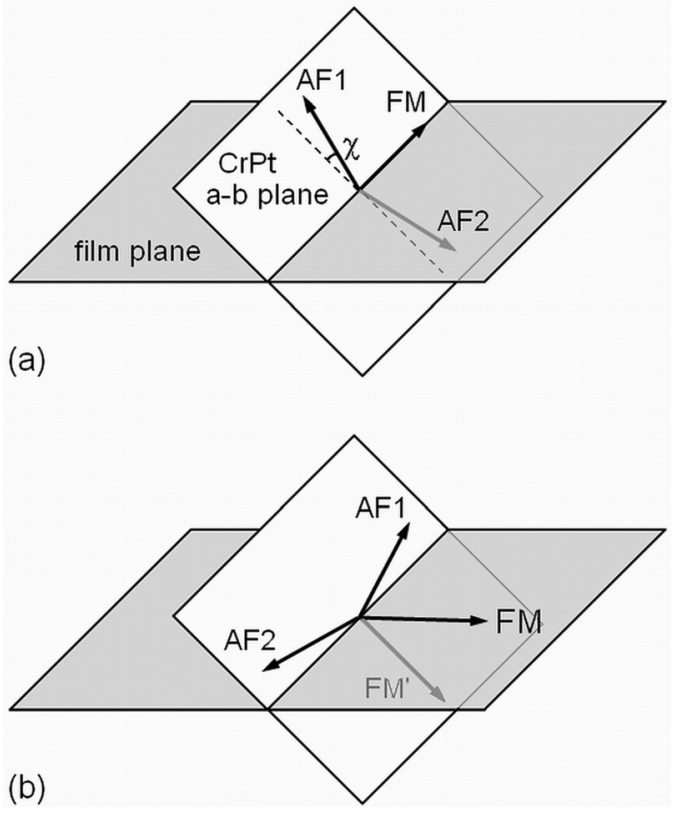

FIG. 5. Effect of spin structure misalignment on $\mathrm{CrPt} / \mathrm{Fe}$ system.

\section{SUMMARY}

In summary, the $L 1_{0}$-ordered antiferromagnetic $\mathrm{CrPt}$ with (001) texture can be formed in samples either deposited or annealed at temperature above $600^{\circ} \mathrm{C}$. The anisotropy from our first principle calculation reproduced the spin structure of $L 1_{0}$ phase $\mathrm{CrPt}$ predicted by neutron spectroscopy. In the present case, experimental result $-0.438 \mathrm{MJ} / \mathrm{m}^{3}$ is significantly smaller than the theoretical prediction of $3.5 \mathrm{MJ} / \mathrm{m}^{3}$, which can be explained as a deviation from Stoner-Wohlfarth behavior.

\section{ACKNOWLEDGMENTS}

This work is supported by Army Research Office under Award No. W911 NF-10-2-0099, National Science Foundation, Materials Research Science and Engineering Center under Award No. NSF-DMR-0820521 and Department of Science and Technology under Award No. SR/NM/NS-20/ 2008. Special thanks to Tom George for all the helpful suggestions regarding the Phase formation.

${ }^{1}$ B. Dai, J. W. Cai, W. Y. Lai, Y. K. An, Z. H. Mai, F. Shen, Y. Z. Liu, and Z. Zhang, Appl. Phys. Lett. 87(9), 092506 (2005).

${ }^{2}$ J. L. Tsai, Y. C. Lin, and C. J. Hsu, J. Phys. D: Appl. Phys. 41(2), 025002 (2008).

${ }^{3}$ D. J. Sellmyer and R. Skomski (Eds.), Advanced Magnetic Nanostructures (Springer, New York, 2009).

${ }^{4}$ A. T. McCallum, P. Krone, F. Springer, C. Brombacher, M. Albrecht, E. Dobisz, M. Grobis, D. Weller, and O. Hellwig, Appl. Phys. Lett. 98(24), 242503 (2011).

${ }^{5}$ S. J. Pickart and R. Nathans, J. Appl. Phys. 34(4), 1203 (1963).

${ }^{6}$ A. Kashyap, R. Skomski, A. K. Solanki, Y. F. Xu, and D. J. Sellmyer, J. Appl. Phys. 95(11), 7480 (2004).

${ }^{7}$ S. D. Mukherjee, D. V. Morgan, and M. J. Howes, J. Electrochem. Soc. 126(6), 1047 (1979).

${ }^{8}$ X.Z. Li, Ultramicroscopy 110(4), 297 (2010).

${ }^{9}$ G. Kresse and D. Joubert, Phys. Rev. B 59(3), 1758 (1999).

${ }^{10} \mathrm{R}$. Skomski and J. M. D. Coey, Permanent Magnetism (Institute of Physics, Bristol, UK, 1999), pp. xi.

${ }^{11}$ R. Skomski, J. Phys.: Condens. Matter 15(20), R841 (2003). 